\title{
Monitoring, Back analysis and Redesign of a Deep excavation Retaining system in marl formation. Case history, Modelling and Interpretation
}

\author{
Chantal Saydi, Youssam Kazan \\ Lebanese University, Faculty of engineering, Roumieh, Lebanon
}

\begin{abstract}
We present in this paper a case study of retaining system of a deep excavation in a marly material in Beirut. The retaining system was redesigned using a new set of mechanical parameter considered more realistic than the set issued from the site investigation study. The new values were issued from back analysis of displacements measurement performed in the same site. The horizontally deflection of the retaining system was measured using three vertical inclinometers. The experimentation results are simulated by numerical calculation using finite elements code.
\end{abstract}

\section{Introduction}

Les formations marneuses abondent dans le territoire libanais. Elles sont étendues dans presque quarante pourcent des zones côtières en surface ou en dessous de la formation sablonneuse érables [1]. Elles sont évidement très sensibles à l'eau et elle s'altèrent facilement quand sont exposées à l'air libre et au changement météorique.

Ces formations sont parfois rocheuses, compactes et classées de bonne qualité. Dans ce cas, leur caractérisation ne prête pas à une confusion en appliquant les règles de la mécanique des roches classique. Mais elles risquent parfois d'avoir des cavités de taille considérables [2], et le traitement de ce problème n'entre pas dans le cadre de ce papier. Dans le cas inverse, ces formations sont tendres, elles seront traitées comme de l'argile raide. La caractérisation sera fait dans les normes de la mécanique des sols.

Cependant le cas intermédiaire prête à une confusion perplexe quand la formation marneuse est dans la limite des roches tendres et les sols durs [3]. En générale, dans le contexte géologique du Liban, cette formation s'agit des bandes et des inclusions rocheuses dans une matrice marneuse très raide. Le prélèvement des échantillons devient une opération délicate et les résultats des essais in situ seront très dispersés.

Les écrans de soutènements de différentes natures, flexibles ou rigides, sont utilisés dans les excavations profondes dans telles formations. Cependant les suivis de comportement des écrans après excavations sont rares, et il est presque impossible de trouver des valeurs publiées [4]. Le seul critère de validations de la méthode de dimensionnement et les paramètres utilisés dans le calcul de ces structures géotechniques est la stabilité de l'excavation lors des travaux d'exécution. Ainsi, les écrans de soutènements, s'ils sont préconisés et exécutés, sont souvent surdimensionnés. Les géotechniciens ne disposent pas de moyens vraiment efficaces pour donner les preuves sur le bon choix de leurs paramètres.

Dans ce pauvre contexte des pratiques géotechniques, le présent papier exposera une étude de cas ainsi qu'une analyse inverse et une modélisation du comportement d'un écran de soutènement réalisé à Sin el fil (banlieue beyrouthin) dans un massif marneux de cette nature. Les paramètres mécaniques, notamment le module d'élasticité utilisé dans le calcul par éléments finis sont issus d'une interprétation de la campagne géotechnique selon les procédures classiques. Les mesures in situ de déformations de l'écran seront analysées et exposées dans le présent papier ainsi que la modélisation sur le logiciel d'élément finis.

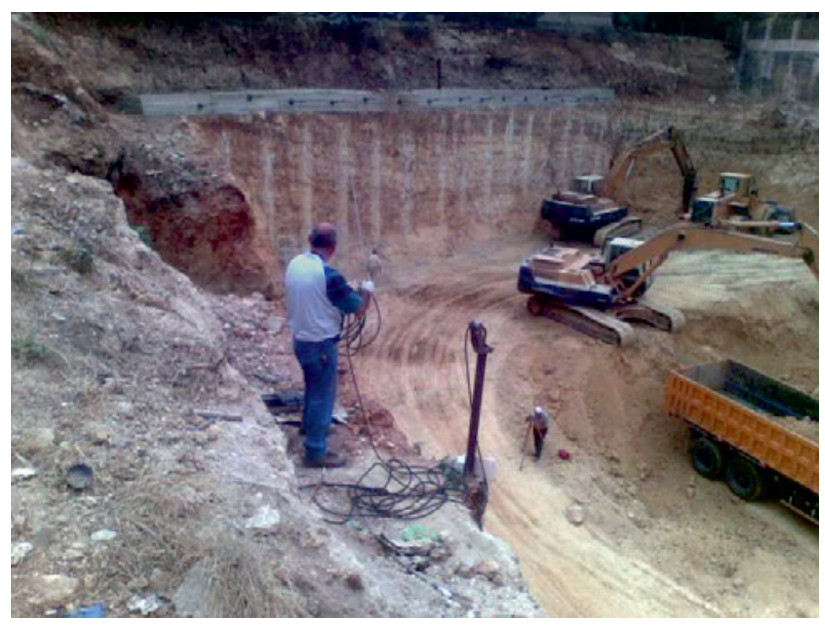

Fig. 1. Vue générale de l'excavation et de l'écran de soutènement 


\section{Description du Projet}

\subsection{Description géométrique}

Il s'agit d'une large excavation qui a la forme d'un trapèze de $2000 \mathrm{~m}^{2}$ de surface. L'excavation est entourée dans les trois cotés par des routes de différentes largeurs. Le niveau des fondations de la future construction est prévu à quinze mètres de profondeur (voir figure 1).

L'absence de constructions approximatives ainsi que le recul exigé par les règlements de l'urbanisme entre la future construction et les routes adjacentes ont permis à l'entrepreneur de procéder à l'excavation sans prévoir un système de soutènement préalable.

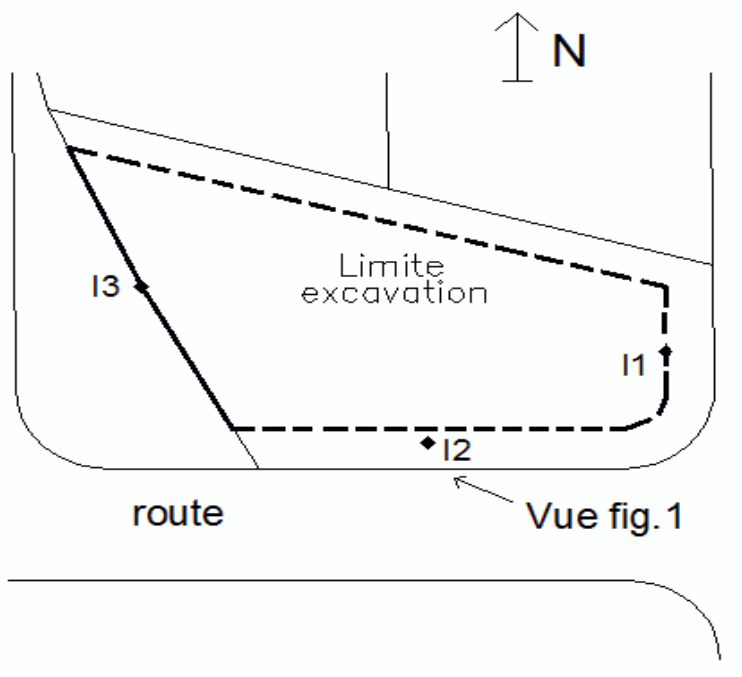

Fig. 2. Plan schématique de l'excavation

\subsection{Contexte géotechnique}

La campagne de reconnaissance de sols et l'étude géotechnique préliminaire ont englobé un nombre total de quatre forages. En résumé, le massif a pu être schématisé par deux couches influentes superposées de marne dont le niveau de séparations de ces couches est variable.

Tableau 1. Description des différentes couches du massif

\begin{tabular}{|c|c|c|}
\hline & Nature & $\begin{array}{l}\text { Action sur la } \\
\text { structure }\end{array}$ \\
\hline $\begin{array}{c}\text { superficielle } \\
2 \mathrm{~m}\end{array}$ & hétérogène & $\begin{array}{l}\text { Pas d'influence vue le } \\
\text { recul disponible }\end{array}$ \\
\hline $\begin{array}{c}\text { Couche active } \\
6 \mathrm{~m}\end{array}$ & Sol marneux & $\begin{array}{l}\text { Action sur écran et } \\
\text { risque d'éboulement }\end{array}$ \\
\hline $\begin{array}{l}\text { Couche } \\
\text { principale } \\
10 \mathrm{~m}\end{array}$ & $\begin{array}{l}\text { Marne raide } \\
\text { avec } \\
\text { inclusions } \\
\text { rocheuses }\end{array}$ & $\begin{array}{l}\text { Action sur l'écran et } \\
\text { réaction buté. } \\
\text { influence sur le } \\
\text { déplacement }\end{array}$ \\
\hline $\begin{array}{c}\text { inférieure } \\
\text { d'épaisseur } \\
\text { indéterminée }\end{array}$ & $\begin{array}{c}\text { Roche } \\
\text { calcaire de } \\
\text { bonne qualité }\end{array}$ & $\begin{array}{l}\text { Considéré comme } \\
\text { substratum }\end{array}$ \\
\hline
\end{tabular}

Les paramètres mécaniques de ces deux couches influentes (appelées dans cet article couche active et couche principale) sont à considérer dans cette étude. A ces deux couches s'ajoutent deux autres couches non influentes: l'une est superficielle, meuble, ayant une épaisseur de deux mètres environ, l'autre est profonde considéré comme substratum rocheux.

\subsection{Paramètres mécaniques du sol.}

Les paramètres mécaniques issus de l'étude géotechnique sont présenté dans la première ligne tu table 2 ci-dessous. Notons que la couche superficielle n'a pas d'influence sur la structure à part son poids propre.

Nous verrons dans la suite que le suivi des mesures de déformations va appuyer la validité des valeurs de la deuxième ligne figurant dans le même tableau par guise de comparaison. D'où la validation de la procédure de construction basée sur la méthode observationnelle.

Tableau 2. Comparaison entre valeurs théoriques admises et valeurs réalistes des paramètres principales des couches

\begin{tabular}{|l|l|c|c|}
\hline & paramètres & $\begin{array}{c}\text { Couche } \\
\text { active }\end{array}$ & $\begin{array}{c}\text { Couche } \\
\text { principale }\end{array}$ \\
\hline Valeurs classiques & $\mathrm{C}(\mathrm{KPa})$ & 30 & 100 \\
déduites de la & $\Phi$ degré & 26 & 28 \\
campagne de & $\gamma(\mathrm{KN} / \mathrm{m} 3)$ & 20 & 21 \\
reconnaissance & $\mathrm{E}(\mathrm{MPa})$ & 20 & 40 \\
\hline Valeurs réalistes & $\mathrm{C}(\mathrm{KPa})$ & 30 & 500 \\
confirmées par & $\Phi$ degré & 34 & 40 \\
suivi et analyse & $\gamma(\mathrm{KN} / \mathrm{m} 3)$ & 20 & 21 \\
inverse & $\mathrm{E}(\mathrm{MPa})$ & 50 & 200 \\
\hline
\end{tabular}

\section{Procédures de construction et suivi}

Dans le but d'optimiser les écrans de soutènement dans le projet, l'ingénieur a décidé de ne pas se fier aux paramètres provenant de la campagne de reconnaissance classique et d'opter à suivre les procédures basées sur la méthode observationnelle.

En effet, l'existence du recul dans les trois cotés, Nord, Est et Sud, a permis d'entreprendre les travaux d'excavation sans risque et sans implanter un écran de soutènement. L'ingénieur a supposé que si un tel écran devient indispensable, il pourra l'exécuter à partir de la profondeur critique. Ce n'était pas le cas de la partie Ouest ou une construction adjacente existe. Dans ce coté, un rideau de pieux de $60 \mathrm{~cm}$ de diamètre a été exécuté entre temps. Il s'agit d'un dimensionnement moyen qui sera complété par le nombre des séries de tirants qui sera déterminé après l'acquisition des données provenant du suivi et de l'analyse inverse.

En résumé, les procédures suivantes ont été adoptées:

1- Décaper le sol des ordures, de la couche superficielle érable et des autres éléments étrangers pour rendre le terrain accessible et plat.

2- Concevoir le soutènement de la partie Ouest et l'emplacement des inclinomètres de forages pour l'acquisition de données utiles.

3- Procéder par l'excavation de la partie centrale du terrain, tout en gardant l'accès au chantier ouverts les zones périphériques. 
4- Procéder par l'exécution de l'écran et des inclinomètres dans chaque coté y compris l'écran et procéder a la lecture initiale des déformations.

5- Poursuivre les travaux d'excavation et la lecture des déformations des inclinomètres dans plusieurs niveaux d'excavation.

6- Interprétation des mesures de déplacement et rémodélisation de l'écran de soutènement. Cette dernière partie porte sur le nombre de tirants.

\subsection{Ecran de soutènement}

Durant l'excavation, il s'est avéré qu'un système de soutènement n'est obligatoire que dans la zone Ouest de l'excavation. L'épaisseur de la couche active de faible cohésion dépassait le six mètres.

Un rideau de pieux en béton armé de soixante centimètres de diamètre, espacés d'un mètre et demi, a été exécuté le long du coté Ouest. Les pieux ont été exécutés après une phase préliminaire d'excavation jusqu'à 2.5 mètres de profondeurs. La profondeur des pieux a été dimensionnée de treize mètres pour prévoir une fiche de trois mètres. Après l'exécution des pieux, on a procédé par l'exécution d'un rang des tirants actifs espacé de $1.5 \mathrm{~m}$ (voir figure 3) Une force de dix tonnes par tirant a été appliquée avant de procéder à la deuxième phase de l'excavation, appelée phase 1 dans le présent article.

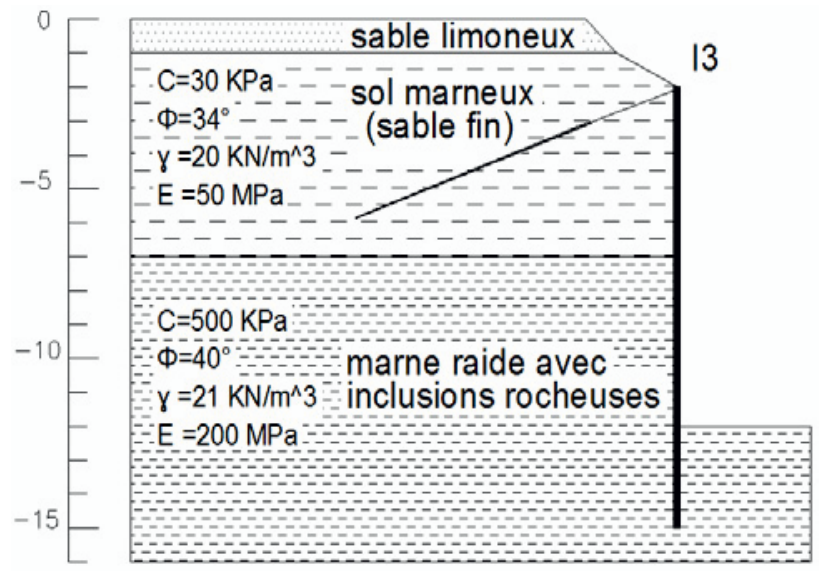

Fig. 3. Coupe typique du massif

Dans ce rideau de pieux un inclinomètre a été placé pour la lecture du déplacement. Vu la nature peu cohésive de la formation active marneuse, le risque d'éboulement de sols entre les pieux n'a pas été réellement présent.

\subsection{Mesures inclinométriques}

Trois inclinomètres de forages ont été installés dans le chantier lors de l'exécution des pieux. Les deux inclinomètres I1 et I 2 ont été placés dans les cotés Est et Sud là où il $\mathrm{n}$ y a pas d'écran. Selon la procédure de déroulement des travaux les résultats de ces inclinomètres vont être utilisés pour dimensionner les autres rangs de tirants dans l'écran de la partie Ouest. En tout, trois campagnes de mesure ont été effectuées y compris les mesures initiales. Les mesures dans chaque inclinomètre ont été effectuées dans une séquence d'un demi-mètre. La figure 2 montre l'emplacement de ces points de mesures.

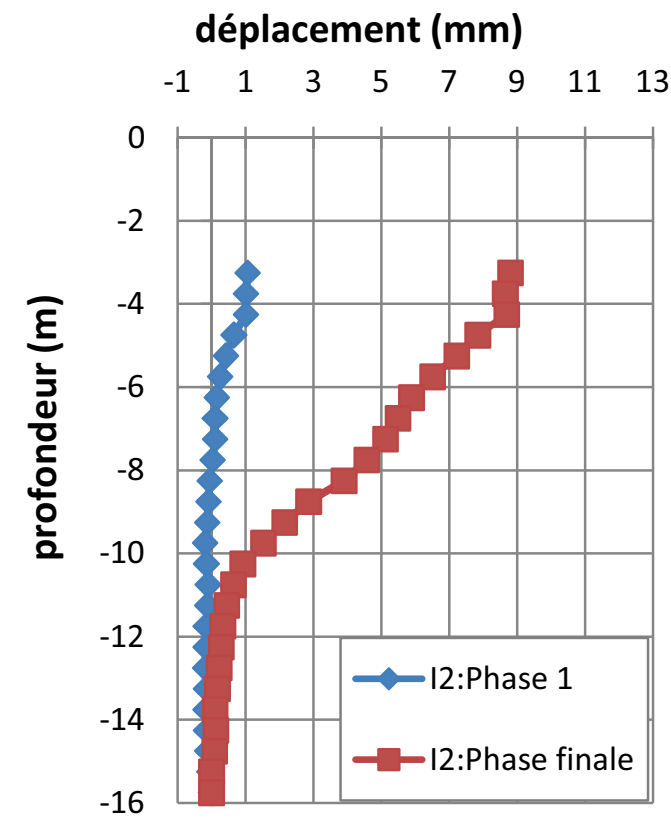

Fig. 4. Mesures de déplacement dans le massif.

Les mesures faites durant la première phase d'excavation a montré un léger déplacement du massif. Le déplacement n'a pas excédé le $1 \mathrm{~mm}$, les travaux d'excavation dans les trois parties périphériques ont été poursuivis sans soutènement. L'excavation a été également poursuivie dans la partie ouest.

Les mesures faites à la fin de la phase d'excavation devant l'inclinomètre $\mathrm{I} 2$ ont permis de proposer les nouveaux paramètre après modélisation du problème sur le logiciel Plaxis.

\section{Modélisation par éléments finis}

Il est évident que le contexte géométrique est loin d'être considéré comme en déformations planes, surtout si l'on considère l'effet du coin. Toutefois, l'utilisation d'un modèle 2-D pourrait être considéré vraisemblable

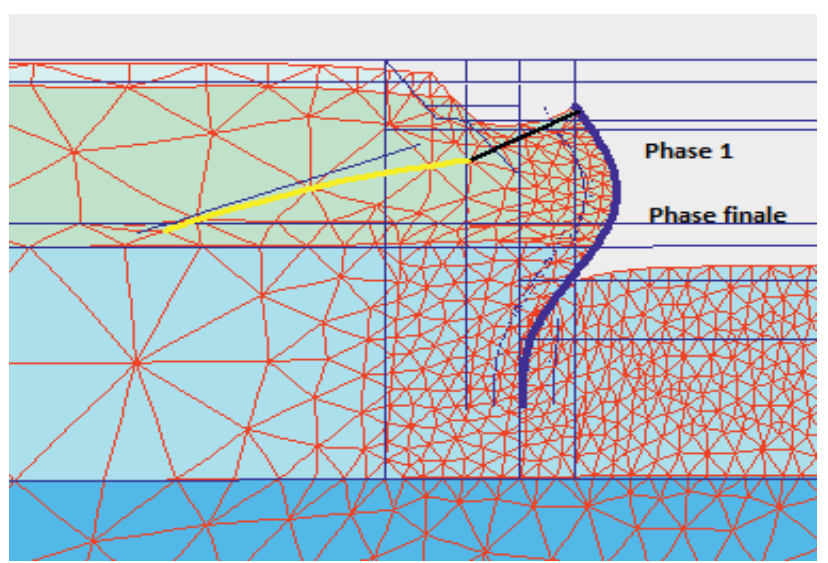

Fig. 5. Coupe typique du maillage par éléments finis 
La simulation des résultats de l'inclinomètre I2 a montré que les paramètres mécaniques du massif sont beaucoup plus grands que les paramètres déduits de la campagne de reconnaissance classique. L'analyse du tableau 2 montre que les valeurs réalistes de la cohésion est du module d'élasticité sont cinq fois plus grandes.

L'allure de la courbe de déplacement lors de la phase final reflète l'influence des deux couches. Le déplacement n'excède pas les $9 \mathrm{~mm}$ bien que la profondeur de l'excavation est de l'ordre de $12 \mathrm{~m}$. La simulation de cette courbe a pu être produite suite à une étude paramétrique non présenté dans ce papier.

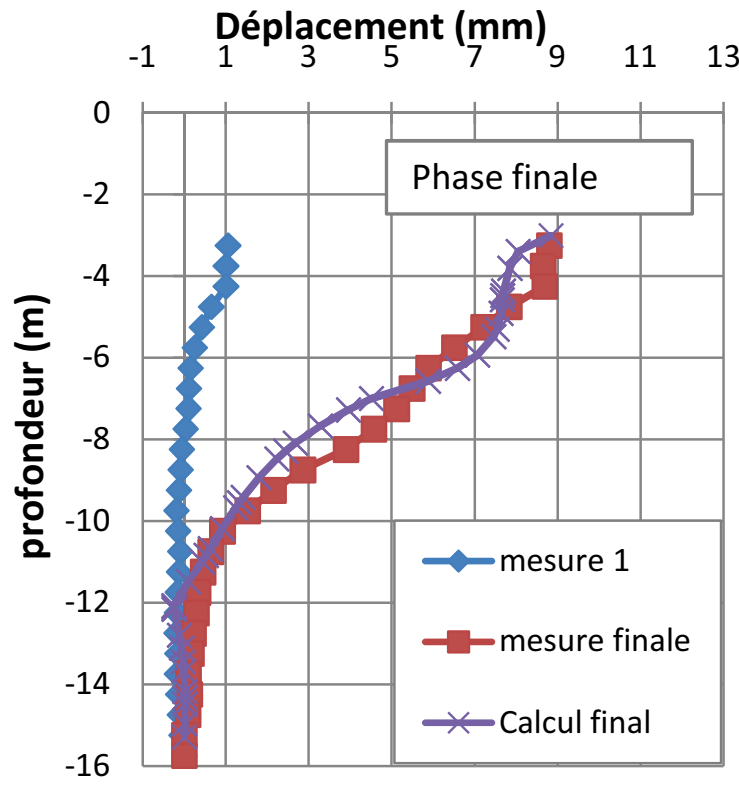

Fig. 6. Simulation de la courbe de déplacement du massif

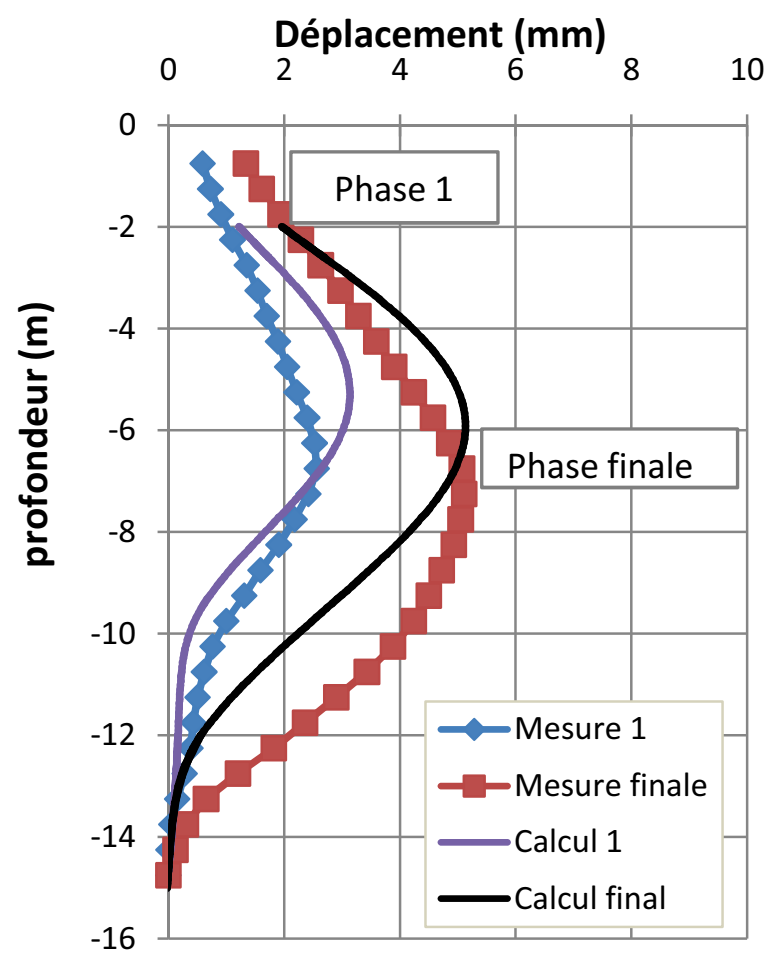

Fig. 7. Simulation de la courbe de déplacement du massif
Les valeurs retenues de l'analyse de l'excavation libre du coté sud sont utilisé dans la section typique de l'écran ou l'inclinomètre $\mathrm{I} 3$ est placé. Le calcul de structure a montré qu'il n'est pas nécessaire d'exécuter des séries de tirants vu la rigidité de massif.

Les courbes dans les figures 7 montrent une bonne simulation des valeurs expérimentales en utilisant les mêmes paramètres. Notons que le module d'élasticité de la couche superficielle était le paramètre mécanique le plus sensible à l'allure des courbes calculées.

\section{Conclusions}

Nous avons pu montrer dans cette étude la validité de la procédure de dimensionnement des écrans de soutènement en utilisant la méthode observationnelle pour déduire les bons paramètres mécanique du sol marneux hétérogène. Les déformations d'une excavation libre (sans écran de soutènement) et d'un écran de soutènement rigide ont été modélisées et présenté dans cet article.

Deux mesures inclinométriques de deux sections différentes ont été faites pour valider le bon dimensionnement. Les paramètres mécaniques ont été déduits de l'interprétation de la courbe de déplacement de l'excavation libre et utilisés pour redimensionner l'écran de soutènement durant les travaux d'excavation.

Bien que l'écran de soutènement lui-même peut être considéré comme simple pour les raisons suivantes :

- un seul rang de tirants actifs a été utilisé pour la stabilité

- seulement deux couches jouent le rôle prépondérant dans les déformations de l'écran.

Cette simplicité a pu focaliser l'attention sur le rôle du module d'élasticité des deux couches marneuses dont le choix de sa valeur a été examiné dans cette modélisation.

Le géotechnicien reconnaît que lors des travaux de modélisation, les courbes calculées n'ont pas été reproduites dès les premières tentatives de calcul. Toutefois, on peut juger de la bonne qualité de modélisation et de la simulation des courbes.

\section{References}

1. Dubertret L. Carte géologique au $50.000^{\mathrm{e}} \mathrm{du}$ Liban. Diffusé par le Ministère des ressources hydrauliques, (1951)

2. Kazan Y., Revue Française de géotechnique, $\mathrm{N}^{\circ} 100$ (2002)

3. Khawlie M. R., Ghannam J. Geological Society of Hong Kong Bulletin $\mathrm{N}^{\circ} 3$ (October 1987)

4. Fawaz A. et al, Actes du Colloque international de géotechniques, Geo-Beyrouth2004. (2004)

5. Houssein A. et al, Symposium international ISP5/pressio2005 (2005) 